\title{
Comprometimento organizacional e qualidade de vida no trabalho para pessoas com e sem deficiência
}

\author{
Maria Nivalda de Carvalho-Freitas - Universidade Federal de São João del-Rei, Minas Gerais, Brasil \\ Liliam Medeiros da Silva - Universidade Federal de São João del-Rei, Minas Gerais, Brasil \\ Suelen Priscila Macedo Farias - Faculdades Integradas Pitágoras de Montes Claros, Minas Gerais, Brasil \\ Marcos Santos de Oliveira - Universidade Federal de São João del-Rei, Minas Gerais, Brasil \\ Raissa Pedrosa Gomes Tette - Universidade Federal de São João del-Rei, Minas Gerais, Brasil
}

\begin{abstract}
Resumo
Esta pesquisa buscou verificar se o comprometimento organizacional e a satisfação com aspectos relacionados à qualidade de vida no trabalho apresentam resultados significativamente diferentes entre pessoas com (PcD) e sem deficiência (PsD). Participaram do estudo 150 trabalhadores, dos quais $75 \mathrm{com}$ e $75 \mathrm{sem}$ deficiência. Os grupos foram emparelhados conforme a função desempenhada e a organização de trabalho. Os instrumentos utilizados foram: Questionário de Comprometimento e de Qualidade de Vida no Trabalho. As respostas foram analisadas quantitativamente. Os resultados permitiram verificar que as PcD têm resultados de satisfação com fatores relacionados ao contexto de trabalho e de comprometimento similares aos das demais pessoas. No entanto, quando possuir uma deficiência se associa a condições sociais de desvantagem (menor renda familiar e primeiro emprego) e ao fato de ser do sexo masculino, as pessoas com deficiência tendem a ter um maior comprometimento instrumental.

Palavras-chave: Comprometimento organizacional, Qualidade de vida no trabalho, Deficiência.
\end{abstract}

\section{Organizational commitment and quality of working life for people with and without disability}

\begin{abstract}
The aim of this research was to verify if organizational commitment and satisfaction with aspects related to quality of working life were different between people with and without disabilities. The sample was constituted of 150 workers, 75 with and 75 without some disability. The groups were paired in function and work organization. The tools used were: Questionnaire about Organizational Commitment and Questionnaire about Satisfaction with Quality of Working Life. The answers were analyzed by quantitative method. The statistical analysis of organizational commitment and satisfaction did not present difference between people with and without disability. The results show that Satisfaction with Quality of working life and commitment are similar between people with and without disability. However, when the disability is associated with conditions of social disadvantage (lower income and first job) and when people with disabilities are males, these people tend to have higher instrumental commitment.

Keywords: Organizational commitment, Quality of working life, Disability.
\end{abstract}

\section{Compromiso con la organización y calidad de vida laboral de personas con y sin discapacidad}

\begin{abstract}
Resumen
Esta investigación buscó verificar si el compromiso con la organización y la satisfacción con aspectos relacionados con la calidad de la vida laboral presentan resultados diferentes entre personas con y sin discapacidad. Participaron 150 trabajadores, 75 con y 75 sin discapacidad. Los grupos fueron emparejados de acuerdo con la función desempeñada y la organización del trabajo. Los instrumentos utilizados fueron: Cuestionario de Compromiso y de Calidad de Vida Laboral. Las respuestas fueron analizadas por método cuantitativo. Los resultados permitieron verificar que las personas con discapacidad tienen resultados de satisfacción con factores relacionados al contexto laboral y de compromiso similares a las demás personas. Sin embargo, cuando la discapacidad se asocia a condiciones de desventaja social (menor ingreso y primer empleo) y al hecho de ser hombre, las personas con discapacidad tienden a tener un mayor compromiso instrumental.

Palabras clave: Compromiso con la organización, Calidad de la vida laboral, Discapacidad.
\end{abstract}

No Brasil, segundo resultados preliminares do censo realizado em 2010 pelo Instituto Brasileiro de Geografia e Estatística (IBGE), existem aproximadamente 46 milhões de pessoas com algum tipo de deficiência, o que representa $24 \%$ da população brasileira. Do total de vínculos empregatícios no país, 0,7\% são formados por pessoas com deficiência (PcD), tendo havido uma elevação de 286,6 mil vínculos empregatícios no ano de 2009 para 306,0 mil em 2010 (Ministério do Trabalho e Emprego, 2010).

Apesar de a inclusão de PcD nas empresas se constituir em um direito garantido por lei, seus resultados ainda têm sido pouco expressivos. Segundo estudo realizado pelo Instituto Ethos (2010), a taxa de participação de $\mathrm{PcD}$ no universo trabalhista não é superior a $1,5 \%$ nas 500 maiores empresas brasileiras pesquisadas. Ademais, a inclusão de $\mathrm{PcD}$ no mercado de trabalho esbarra em grandes dificuldades para se afirmar em relação, principalmente, do descrédito dos empregadores em relação às possibilidades de trabalho das PcD e das alegações de baixa qualificação dessa população (Maia, Camino C., \& Camino L., 2011; Ribeiro \& Carneiro, 2009; Tanaka \& Manzini, 2005). Além disso, vários estudos identificam as dificuldades 
geradas pelo contexto e condições de trabalho para as pessoas com deficiência (Butterfield \& Ramseur, 2004; Gartrell, 2010; Jolly, 2000; Opini, 2010; Shier, Grahan \& Jones, 2009; Stone \& Colella, 1996; Stone-Romero, Stone \& Lukaszewski, 2006; Wehbi \& El-Lahib, 2007; Zolna, Sanford, Sabata \& Goldthwaite, 2007).

Por outro lado, as pesquisas que focam nas PcD empregadas, no mercado competitivo têm mostrado que elas possuem níveis elevados de satisfação em relação aos diversos aspectos relacionados à qualidade de vida no trabalho, sendo o aspecto referente às possibilidades de carreira e progressão dentro das organizações o único fator a apresentar resultados de insatisfação (Almeida, Carvalho-Freitas \& Marques, 2009; Carvalho-Freitas, 2009; Carvalho-Freitas, Marques, \& Almeida, 2009; Barbosa-Gomes, 2009). Uma questão que aparece recorrentemente nas pesquisas que se debruçam sobre a inserção de $\mathrm{PcD}$ no mercado de trabalho diz respeito ao vínculo que as PcD mantêm com o trabalho. Os resultados dessas investigações mostram divergências: alguns resultados indicam que os gestores acreditam na incapacidade das PcD para o trabalho e que elas usam o fato de possuírem deficiência para não trabalhar adequadamente (Nascimento, Damasceno, \& Assis, 2011; Ribeiro \& Carneiro, 2009). Outros estudos indicam que os gestores pensam que, pelas maiores dificuldades em se conseguir um emprego, as PcD são mais comprometidas com o trabalho; ou, então, os gestores apresentam dúvidas em relação ao vínculo das PcD com o trabalho, isto é, não sabem se o compromisso que elas têm com o trabalho se diferencia do das demais pessoas (Carvalho-Freitas, 2009, Carvalho-Freitas, \& Marques, 2010).

Em pesquisa realizada com $\mathrm{PcD}$ empregadas em instituições financeiras (bancos) de um grande centro urbano (Carvalho-Freitas e cols., 2009), foi verificado que o tipo predominante de comprometimento estabelecido por elas em relação à organização em que trabalhavam era o de base afetiva, isto é, as $\mathrm{PcD}$ que fizeram parte da investigação permaneciam na organização em que trabalhavam porque assim o desejavam, e não por se sentirem gratas à organização ou por não verem outras possibilidades de trabalho. No entanto, essa pesquisa não comparou os resultados das $\mathrm{PcD}$ com pessoas que não tinham deficiência, o que não permitiu verificar se o vínculo com o trabalho das PcD era semelhante ou não ao das demais pessoas. Nenhuma pesquisa comparativa entre pessoas com e sem deficiência no que se refere ao comprometimento organizacional foi identificada na literatura nacional ou internacional.

Esse cenário levou à seguinte interrogação: será que os resultados de comprometimento organizacional dependem do fato de ser pessoa com deficiência? Será que pessoas com e sem deficiência apresentam resultados de comprometimento e satisfação diferentes em situações semelhantes de trabalho? Assim, o objetivo desta pesquisa foi verificar se o fato de se possuir uma deficiência é realmente um fator distintivo nas relações de trabalho ou se, em condições similares, pessoas com e sem deficiência apresentam características semelhantes de comprometimento organizacional e satisfação com o trabalho.

Os estudos sobre comprometimento organizacional tiveram um intenso crescimento a partir do final da década de 1970. As pesquisas nessa área são marcadas pela diversidade de definições, resultando numa grande variedade de instrumentos de medida. Rodrigues e Bastos (2009) afirmam que os pesquisadores estão envolvidos em uma ampla agenda de pesquisa no intuito de superar divergências conceituais e empíricas em estudos sobre o tema. Esses autores encontraram diferentes definições de comprometimento organizacional, sendo estas classificadas em dois tipos de vínculos e quatro características básicas, que são vínculo ativo cujas características são o engajamento e empenho extra, e identificação e afeto; e o vínculo passivo, que possui como características permanência e instrumentalidade e relação de troca.

Embora não haja consenso quanto ao conceito de comprometimento organizacional, este estudo utilizou a ideia referendada por Siqueira e Gomide Júnior (2004), que consiste no comprometimento organizacional como vínculo que é estabelecido entre o trabalhador e a organização de trabalho. Bastos, Brandão e Pinho (1997) acrescentam que o comprometimento se assemelharia à disposição. Assim, esse construto é tomado como um estado, caracterizado por sentimentos ou reações afetivas, do indivíduo para com a organização.

Bastos e cols. (1997) identificam duas bases psicológicas de comprometimento na atualidade: a base afetiva - que se sustenta no pressuposto de que o indivíduo se identifica e nutre sentimentos positivos ou negativos em relação à organização de trabalho da qual faz parte; e a base cognitiva - que se funda na premissa de que o indivíduo desenvolve um conjunto de crenças sobre recompensas, sustentadas nas relações de troca com a organização. $\mathrm{O}$ comprometimento organizacional, portanto, seria construído a partir dessas duas bases psicológicas (afetiva e cognitiva).

Dentre os diversos modelos de comprometimento organizacional, o de maior utilização entre os pesquisadores é o modelo proposto por Allen e Meyer (1996). Ao criarem esse modelo de análise, esses autores consideraram três componentes: 
Comprometimento afetivo: possui base psicológica afetiva e se refere ao envolvimento do indivíduo com a organização, bem como com a introjeção dos valores da empresa aos seus próprios valores. Assim, o indivíduo permanece na organização porque deseja. Comprometimento instrumental: possui base cognitiva e é ligado ao reconhecimento dos custos associados à saída da organização, ou seja, o colaborador reconhece que, se abandonar a empresa, perderá investimentos nela feitos. Comprometimento normativo: possui base cognitiva, relacionado à obrigação moral para com a empresa, visto que recebe benefícios da organização e por isso se sente no dever de atuar reciprocamente. Segundo Allen e Meyer (1996), os dois últimos tipos de vínculos são de base cognitiva. Esses componentes do comprometimento são coexistentes, sendo possível uma mesma pessoa possuí-los, simultaneamente, em graus diferentes.

Diferentes reações atitudinais de comprometimento surgem a partir da maior ou menor predominância de um ou outro tipo de vínculo. Isso significa que trabalhadores com altos escores de comprometimento afetivo, provavelmente, são pessoas que possuem maior motivação para contribuir com o desempenho da organização, apresentam baixo turnover, menor absenteísmo e mantêm uma prática maior de comportamentos de cidadania organizacional (Rego, Cunha, \& Souto, 2007). De maneira diferente, segundo esses pesquisadores, as pessoas que possuem altos níveis de comprometimento instrumental, possivelmente, não se disponibilizam a dar à organização mais do que aquilo a que estão obrigadas e, assim, permanecem na instituição porque precisam. Por outro lado, aqueles colaboradores com índices maiores de comprometimento normativo tendem a adotar comportamentos positivos, porém sem a mesma implicação e ânimo que os produzidos pelo comprometimento afetivo.

Conforme Bastos (1994), os vínculos do trabalhador com a organização estão relacionados a múltiplos aspectos relativos ao contexto de trabalho. Por contexto de trabalho entendem-se as fontes ou origens das experiências que geram satisfação, por exemplo, salário, relação com chefias e colegas, condições de trabalho, possibilidade de promoção etc. Nesse sentido, os estudos de Qualidade de Vida no Trabalho (QVT) trazem uma possibilidade de avaliar a satisfação do trabalhador com esses fatores de contexto que podem impactar os vínculos com a organização. Várias pesquisas têm investigado a satisfação relacionada aos aspectos de QVT (Honório, Marques, \& Melo, 2001; Martins \& Santos, 2006; Vasconcelos, 2001). Esses estudos mostram que os modelos teóricos de QVT mais utilizados e validados, dentro do contexto nacional, são os de Hackman e Oldham (1975) e Walton (1973).

Optou-se, neste estudo, por trabalhar a QVT a partir da perspectiva de Walton (1973), pois esse autor buscou identificar elementos nas organizações que pudessem garantir a humanização das relações de trabalho, considerando aspectos relevantes quando se fala em minorias, como a dimensão dos direitos do trabalhador. Ele propõe oito fatores relacionados ao construto: Compensação justa e adequada (remuneração): expressa equidade interna (comparação com os colegas) e externa (comparação com o mercado) em termos de salário direto e indireto (benefícios). Condições de segurança e saúde no trabalho (condições de trabalho): refere-se às condições reais de trabalho oferecidas ao colaborador para que o mesmo possa executar suas tarefas. Esta categoria envolve as questões referentes à segurança, saúde, jornada e carga de trabalho, equipamentos disponibilizados para a execução do trabalho e ambiente saudável (preservação da saúde do trabalhador). Uso e desenvolvimento de capacidades: implica o aproveitamento do talento humano, ou seja, a aplicação do capital intelectual dentro da organização. Significa autonomia, uso de diversas habilidades e participação no processo total de trabalho. Oportunidade de crescimento e segurança (oportunidades de crescimento profissional): significa a possibilidade de crescimento, desenvolvimento e manutenção do trabalho dos empregados dentro da organização. Integração social na organização: significa ausência de preconceitos, equidade de oportunidades e cultivo ao bom relacionamento dentro da instituição. Constitucionalismo (direitos na instituição): diz respeito ao cumprimento das normas e procedimentos relacionados às leis e direitos trabalhistas. Trabalho $e$ espaço total da vida (equilíbrio trabalho e vida): refere-se à possibilidade de equilíbrio entre o tempo e o ânimo dedicados à empresa e aos demais espaços da vida. Relevância do trabalho na vida (relevância do trabalho): diz respeito à percepção do empregado em relação à imagem da empresa e à responsabilidade social da organização.

Estudos indicam que a satisfação é entendida como uma forma de vínculo afetivo e considerada uma variável correlata ao comprometimento (Mathieu \& Zajac, 1990; Meyer, Stanley, Herscovitch, \& Topolnytsky, 2002; Siqueira \& Gomide Jr., 2004). Por outro lado, esses estudos consideram que os comprometimentos afetivo, normativo ou instrumental podem ser, cada um à sua maneira, dependentes de antecedentes como características individuais e/ou experiências de trabalho e de socialização, alternativas de emprego e investimento no trabalho. Em função disso, o presente estudo investigou as correlações entre 
comprometimento organizacional e satisfação com fatores de QVT; e as relações de dependência entre comprometimento e características sociodemográficas, visando ampliar as possibilidades de discussão do vínculo com a organização introduzindo a variável condição de deficiência.

As principais hipóteses do estudo foram: 1) Não existe diferença significativa entre os resultados de pessoas com e sem deficiência, em situações similares de trabalho, no que se refere ao comprometimento e satisfação com fatores relacionados à QVT; 2) existe correlação positiva significativa entre comprometimento afetivo e satisfação com fatores de qualidade de vida no trabalho entre pessoas com e sem deficiência; 3) existe relação de dependência entre tipo de comprometimento e variáveis sociodemográficas; e 4) não existe relação de dependência entre tipo de comprometimento e condição de deficiência.

\section{Método}

A pesquisa possui uma natureza descritiva, relacionada ao esforço de caracterização dos resultados de comprometimento organizacional e satisfação com aspectos relacionados à qualidade de vida no trabalho de pessoas com e sem deficiência. Também possui natureza explicativa, pois buscará identificar se existem relações entre os construtos analisados (comprometimento organizacional e qualidade de vida no trabalho) e se os resultados de comprometimento dependem de fatores sociodemográficos e da condição de deficiência. A pesquisa foi submetida ao comitê de ética da Instituição, tendo recebido parecer favorável para sua realização.

\section{Participantes}

Formando dois grupos emparelhados em relação ao cargo ocupado e à organização de trabalho, participaram da pesquisa 75 pessoas com deficiência (PcD) e 75 pessoas sem deficiência (PsD). O critério de inclusão na amostra foi o pareamento em relação às funções executadas e a organização em que trabalhavam.

A amostra de PcD foi assim constituída: 56\% eram do sexo masculino e $44 \%$ do sexo feminino, cuja média de idade era de 32 anos e o desvio padrão era de nove anos. Além disso, $62,7 \%$ afirmaram já ter trabalhado de maneira informal e 37,3\% trabalharam no mercado formal. Em relação ao tipo de deficiência da população pesquisada, 9,3\% das pessoas possuíam deficiência visual, 28\% deficiência auditiva e 62,7\% deficiência física.

Já na amostra constituída por PsD, 49,3\% das pessoas eram do sexo masculino e $50,7 \%$ do sexo feminino, a média de idades destes era de 33 anos e o desvio padrão era dez anos. Dessa amostra, 68\% afirmaram já ter trabalhado sem carteira assinada; $68 \%$ alegaram ser esse seu primeiro emprego formal e $32 \%$ dos respondentes disseram já ter trabalhado formalmente antes do atual emprego.

Das pessoas que participaram da pesquisa, grande parte se considerou de cor parda: 46,7\% das PcD e 48,7\% das PsD. Em relação ao estado civil, $57,3 \%$ das $\mathrm{PcD}$ e $45,3 \%$ das $\mathrm{PsD}$ eram solteiras. Em relação à classe socioeconômica familiar, a maioria das pessoas relatou ter renda familiar de um a dois salários mínimos: $40 \%$ das PcD e $29,3 \%$ das PsD. Foi verificado que $13,3 \%$ das $\mathrm{PsD}$ possuía uma renda familiar de dez a 20 salários mínimos, e que apenas $5,3 \%$ das PcD possuía uma renda familiar similar a essa quantia. O Ensino Médio completo correspondeu ao grau de escolaridade de praticamente metade da amostra (41,3\% das PcD e 49,3\% das PsD).

A partir da análise dos dados da população estudada, verificou-se que houve certa equivalência entre os dois grupos no item referente ao número de empregos anteriores, sendo que $42 \%$ das $\mathrm{PcD}$ e $44,2 \%$ das PsD alegaram ter trabalhado apenas em outro emprego. Em relação ao tempo de trabalho anterior, as pessoas com e sem deficiência $(48,1 \%$ e $35,8 \%$, respectivamente) afirmaram que tiveram de um a cinco empregos anteriores. A maior parte dos entrevistados (55,4\% e $38,7 \%$, respectivamente) alegou ter de cinco a dez anos de trabalho na organização em que atualmente trabalham. A respeito do nível salarial total, $40 \%$ das $\mathrm{PcD}$ afirmaram receber até um salário mínimo, enquanto $44 \%$ das pessoas sem deficiência afirmaram receber a mesma quantia. A amostra foi constituída por pessoas com e sem deficiência. Não foram contempladas na presente pesquisa pessoas com deficiência intelectual em virtude dos instrumentos utilizados para a pesquisa (questionários).

\section{Local}

A realização do estudo ocorreu em oito cidades de Minas Gerais, sendo a capital do estado, com cerca de 2,3 milhões de habitantes, e sete cidades do interior, com populações variando entre 600 mil habitantes, a maior cidade, e 23 mil habitantes, a menor cidade. As atividades das organizações que concentraram a maior parte da amostra foram comércio (56\%) e educação $(11,9 \%)$. As demais pessoas pertenciam a distintos tipos de organizações.

\section{Instrumentos}

Questionário Sociodemográfico contendo questões relativas ao sexo, cor, idade, classe socioeconômica; dados referentes aos aspectos 
funcionais: trabalhos anteriores, se no mercado formal ou informal; e no trabalho atual, como salário e tempo de permanência na atual organização.

Questionário de comprometimento Organizacional (Rego e cols., 2007), utilizando-se escala de 1 (discordo totalmente) a 5 (concordo totalmente), contendo 11 afirmativas contemplando os três componentes de comprometimento propostos por Allen e Meyer (1996) e usado como referência na presente pesquisa. Resultados de estudo de análise fatorial confirmatória do instrumento indicam validade satisfatória, com índices de consistência interna (alfas de Crombach) entre 0,76 e 0,86 (Rego e cols., 2007).

Inventário de Qualidade de Vida no Trabalho adaptado a partir do modelo proposto por Walton (Carvalho-Freitas \& Marques, 2009), utilizando-se escala de 1 (totalmente insatisfeito) a 6 (totalmente satisfeito), contendo 45 afirmativas relacionadas aos oito fatores de QVT (Walton, 1973). Resultados de estudo de análise da precisão do instrumento, por meio do método de alfa de Crombach, indicaram bons índices de consistência interna, entre 0,81 e 0,95 (Carvalho-Freitas, 2007).

\section{Procedimento de coleta de dados}

As pessoas com deficiência foram contactadas e informadas sobre os objetivos da pesquisa. Foi identificado se havia pessoa sem deficiência que executava a mesma função na organização. Após o consentimento dos participantes, foram aplicados os questionários. A aplicação dos instrumentos foi realizada de formas diferentes em função da realidade das organizações e das PcD: a maioria foi presencial e as pesquisadoras davam as instruções gerais e aguardavam a leitura e o preenchimento dos questionários; em alguns casos, em função do tipo de deficiência (por exemplo, deficiência visual), os questionários foram preenchidos pelas pesquisadoras; e, em outros casos, foi necessária a utilização da língua brasileira de sinais (LIBRAS) para o contato e as explicações gerais sobre o questionário. Quando as pessoas não eram autorizadas a responder aos questionários no local de trabalho, foram feitas as explicações e deixados os questionários com elas, que os devolviam no dia seguinte para as pesquisadoras. Houve, ainda, alguns casos em que as explicações, o encaminhamento e a devolução dos questionários foram por e-mail. Nesses casos, era interrogado se haviam tido dúvidas ou questões sobre as quais quisessem conversar.

\section{Análise dos dados}

A análise dos dados envolveu a utilização de alguns procedimentos e técnicas estatísticas. Utilizou-se estatística descritiva para análise dos dados sociodemográficos. A avaliação de possíveis diferenças entre as médias dos resultados de pessoas com e sem deficiência quanto ao comprometimento organizacional e satisfação com aspectos de QVT foi realizada utilizando-se teste $t$ para amostras independentes. Foi utilizada a análise de correlação de Pearson com o intuito de verificar a ocorrência de correlações (direção e intensidade das associações) entre os fatores dos dois construtos analisados. Realizaram-se, ainda, análises de regressão linear múltipla. Os modelos de regressão foram construídos por meio do método stepwise, no qual a seleção de entrada das variáveis preditivas é feita estatisticamente. A escolha desse método de regressão se justifica, pois não há consenso na literatura a respeito da ordenação causal das variáveis preditivas do comprometimento (Mathieu \& Zajac, 1990; Meyer, Stanley, Herscovitch, \& Topolnytsky, 2002). Para cada análise realizada, foi calculado o índice de Durbin-Watson (DW), que testa se os resíduos adjacentes ao plano de regressão são correlacionados e corrobora a hipótese de distribuição normal dos dados quando seus valores são próximos ou superiores a 2. Além disso, foi feito o teste Kolmogorov-Smirnov dos resíduos, visando confirmar a distribuição normal dos modelos de regressão.

\section{Resultados}

Para avaliar se existia diferença significativa entre as médias dos grupos de pessoas com e sem deficiência foi utilizado o teste $t$ para amostras independentes. A análise dos dados permitiu verificar que o comprometimento afetivo foi o componente possuidor de uma média maior, tanto entre o grupo de $\mathrm{PcD}$ quanto entre o grupo de $\mathrm{PsD}$ (Tabela 1). Em relação à satisfação com os fatores de qualidade de vida no trabalho das pessoas com e sem deficiência, foi possível observar, de acordo com a Tabela 1, que as médias dos fatores também foram bastante similares entre os dois grupos.

A análise comparativa de médias (teste $t$ ) não indicou diferença estatística entre os grupos de $\mathrm{PcD}$ e PsD em relação aos resultados de comprometimento e de satisfação com os fatores de QVT. Tendo como referência esse resultado, foram analisadas as possíveis correlações entre o comprometimento e os fatores de QVT de todos os participantes da pesquisa, tendo sido identificadas correlações de fraca a moderada entre a maioria dos fatores de comprometimento e QVT, conforme Tabela 2. 
Tabela 1. Resultados do Teste t de Comprometimento e Satisfação de Pessoas com e sem Deficiência

\begin{tabular}{|c|c|c|c|c|c|}
\hline Fatores & Classificação & Média & Desvio Padrão & $t$ & $p$ \\
\hline \multirow{2}{*}{ Comprometimento afetivo } & PsD & 4,009 & 0,907 & \multirow{2}{*}{$-1,132$} & \multirow{2}{*}{0,259} \\
\hline & PcD & 4,167 & 0,796 & & \\
\hline \multirow{2}{*}{ Comprometimento normativo } & PsD & 2,678 & 1,248 & \multirow{3}{*}{0,212} & \multirow{3}{*}{0,832} \\
\hline & $\mathrm{PcD}$ & 2,638 & 1,050 & & \\
\hline \multirow{2}{*}{ Comprometimento instrumental } & PsD & 2,534 & 1,048 & & \\
\hline & PcD & 2,796 & 1,190 & \multirow[t]{2}{*}{$-1,433$} & \multirow[t]{2}{*}{0,154} \\
\hline \multirow{2}{*}{ Remuneração } & PsD & 3,281 & 1,045 & & \\
\hline & PcD & 3,441 & 1,032 & \multirow[t]{2}{*}{$-0,943$} & \multirow[t]{2}{*}{0,347} \\
\hline \multirow{2}{*}{ Condições de trabalho } & $\mathrm{PsD}$ & 3,760 & 0,861 & & \\
\hline & PcD & 3,840 & 1,034 & \multirow[t]{2}{*}{$-0,510$} & \multirow[t]{2}{*}{0,610} \\
\hline \multirow{2}{*}{ Uso e desenvolvimento de capacidades } & PsD & 3,980 & 0,706 & & \\
\hline & PcD & 4,041 & 1,024 & \multirow[t]{2}{*}{-0423} & \multirow[t]{2}{*}{0,673} \\
\hline \multirow{2}{*}{ Oportunidades crescimento profissional } & $\mathrm{PsD}$ & 3,700 & 0,852 & & \\
\hline & $\mathrm{PcD}$ & 3,564 & 1,080 & \multirow[t]{2}{*}{0,853} & \multirow[t]{2}{*}{0,395} \\
\hline \multirow{2}{*}{ Integração social na organização } & PsD & 4,304 & 0,759 & & \\
\hline & $\mathrm{PcD}$ & 4,280 & 0,898 & \multirow[t]{2}{*}{0,176} & \multirow[t]{2}{*}{0,860} \\
\hline \multirow{2}{*}{ Direitos na instituição } & PsD & 3,950 & 0,779 & & \\
\hline & $\mathrm{PcD}$ & 4,085 & 1,018 & \multirow[t]{2}{*}{$-0,911$} & \multirow[t]{2}{*}{0,364} \\
\hline \multirow{2}{*}{ Equilíbrio trabalho e vida } & PsD & 3,627 & 0,767 & & \\
\hline & PcD & 3,641 & 1,160 & \multirow[t]{2}{*}{$-0,090$} & \multirow[t]{2}{*}{0,928} \\
\hline \multirow{2}{*}{ Relevância do trabalho } & PsD & 4,210 & 0,648 & & \\
\hline & $\mathrm{PcD}$ & 4,432 & 0,888 & $-1,742$ & 0,084 \\
\hline
\end{tabular}

Tabela 2. Correlação de Pearson entre comprometimento e satisfação no trabalho

\begin{tabular}{lccc}
\hline \multicolumn{1}{c}{ Fatores } & \multicolumn{3}{c}{ Comprometimento } \\
\cline { 2 - 4 } & Afetivo & Normativo & Instrumental \\
\hline Remuneração & $0,506^{* *}$ & $0,382^{* *}$ & $-0,054$ \\
Condições de trabalho & $0,526^{* *}$ & $0,348^{* *}$ & $-0,096$ \\
Uso e desenvolvimento de capacidades & $0,427^{* *}$ & $0,325^{* *}$ & $-0,115$ \\
Oportunidades crescimento profissional & $0,476^{* *}$ & $0,411^{* *}$ & $-0,152$ \\
Integração social na organização & $0,400^{* *}$ & $0,224^{* *}$ & $-0,189^{*}$ \\
Direitos na instituição & $0,459^{* *}$ & $0,268^{* *}$ & $-0,117$ \\
Equilíbrio trabalho e vida & $0,245^{* *}$ & $0,181^{*}$ & $-0,154$ \\
Relevância do trabalho & $0,450^{* *}$ & $0,200^{*}$ & $-0,297^{* *}$ \\
\hline
\end{tabular}

* Correlação significativa $p<0,05$

** Correlação significativa $p<0,01$

A fim de identificar as contribuições específicas de variáveis para a predição do comprometimento, foram utilizadas análises de regressão (método stepwise). Foram analisadas três possibilidades de explicação preditiva, tendo como variáveis dependentes: primeiro, o comprometimento afetivo; depois, o normativo; e, por último, o instrumental. Para cada tipo de comprometimento foram realizadas duas análises de regressão. $\mathrm{Na}$ primeira foram utilizadas as variáveis sociodemográficas: sexo, idade, estado civil (vive com parceiro ou não), escolaridade (até ensino médio ou acima), classe econômica (até 3 salários mínimos ou acima), se primeiro emprego, tempo na função atual (menor que 1 ano, de 1 a 5 anos ou acima de 5 anos) e salário atual. $\mathrm{Na}$ segunda, foi introduzida a variável condição de deficiência como possível variável explicativa no conjunto de variáveis identificadas. Assim, a diferença no percentual explicado $\left(\mathrm{R}^{2}\right.$ ajustado), quando se compara a segunda etapa da análise com a primeira, indica o quanto a variável condição de deficiência acrescenta ao modelo explicativo do comprometimento, para além do que as variáveis sociodemográficas já explicaram.

As variáveis associadas à satisfação com a QVT não foram utilizadas para a construção dos modelos de regressão, pois a satisfação é um dos diversos conceitos que abordam a afetividade no ambiente de trabalho, sendo considerada uma forma de vínculo afetivo do indivíduo com o trabalho (Siqueira \& Gomide Jr., 
2004), e uma variável correlata ao comprometimento (Meyer e cols., 2002)

Os resultados das análises de regressão para o comprometimento afetivo não apresentaram distribuição normal. O teste de Kolmogorov-Smirnov dos resíduos apresentou, respectivamente, $p=0,026$, para o modelo que utilizou variáveis sociodemográficas; e $p=0,014$, inserindo, além das variáveis sociodemográficas a variável condição de deficiência, confirmando a hipótese de ausência de normalidade dos resíduos.

Para a análise de regressão referente ao comprometimento normativo, foi identificada a normalidade dos modelos de explicação. O teste de
Kolmogorov-Smirnov apresentou, respectivamente, bloco 1 com $p=0,327$; bloco 2 com $p=0,295$, confirmando a hipótese de normalidade dos resíduos, o que respalda os resultados da regressão.

Os resultados das análises de regressão para o comprometimento normativo são apresentados na Tabela 3. Como se podem observar, apenas duas variáveis sociodemográficas têm poder explicativo sobre o comprometimento normativo. No entanto, explicam apenas $4,9 \%\left(\mathrm{R}^{2}\right.$ ajustado $\left.=0,049\right)$ da variância dos resultados. Quando a variável condição de deficiência é inserida, verifica-se um pequeno decréscimo no poder explicativo do modelo $\left(\mathrm{R}^{2}\right.$ ajustado $=0,043)$.

Tabela 3. Resultados da análise de regressão para comprometimento normativo

\begin{tabular}{lccccc}
\hline \multicolumn{1}{c}{ Bloco 1 } & & & \\
\hline \multicolumn{1}{c}{ Variáveis preditivas } & Beta & $\begin{array}{c}\text { Erro } \\
\text { padrão }\end{array}$ & $\begin{array}{c}\text { Beta } \\
\text { padronizado }\end{array}$ & $t$ & $p$ \\
\hline Constante & 2,607 & 0,237 & & 10,997 & $0,000^{*}$ \\
Tempo emprego atual (de 1 a 5 anos) & $-0,452$ & 0,224 & $-0,197$ & $-2,014$ & $0,046^{*}$ \\
Tempo emprego atual (acima de 5 anos) & 0,086 & 0,260 & 0,033 & 0,330 & 0,742 \\
Classe econômica & 0,376 & 0,197 & 0,158 & 1,911 & 0,058 \\
\hline
\end{tabular}

$\mathrm{R}^{2}$ ajustado $=0,049 ; \mathrm{F}(3,146)=3,587 ; p=0,015 ; \mathrm{DW}=1,804 . /$ Nota: ${ }^{*} p<0,05$

\begin{tabular}{lccccc}
\hline \multicolumn{1}{c}{ Bloco 2 } & & & \\
\hline \multicolumn{1}{c}{ Variáveis preditivas } & Beta & $\begin{array}{c}\text { Erro } \\
\text { padrão }\end{array}$ & $\begin{array}{c}\text { Beta } \\
\text { padronizado }\end{array}$ & $t$ & $p$ \\
\hline Constante & 2,599 & 0,251 & & 10,343 & $0,000^{*}$ \\
Tempo emprego atual (de 1 a 5 anos) & $-0,454$ & 0,226 & $-0,198$ & $-2,007$ & $0,047^{*}$ \\
Tempo emprego atual (acima de 5 anos) & 0,087 & 0,261 & 0,034 & 0,335 & 0,738 \\
Classe econômica & 0,375 & 0,198 & 0,158 & 1,897 & 0,060 \\
Condição de deficiência & 0,019 & 0,187 & 0,008 & 0,103 & 0,918 \\
\hline
\end{tabular}

$\mathrm{R}^{2}$ ajustado $=0,043 ; \mathrm{F}(3,146)=3,587 ; p=0,015 ; \mathrm{DW}=1,804 . \quad$ / Nota: ${ }^{*} p<0,05$

Tabela 4. Resultados da Análise de Regressão para Comprometimento Instrumental

\begin{tabular}{|c|c|c|c|c|c|}
\hline \multicolumn{6}{|c|}{ Bloco 1} \\
\hline Variáveis preditivas & Beta & $\begin{array}{c}\text { Erro } \\
\text { Padrão }\end{array}$ & $\begin{array}{c}\text { Beta } \\
\text { padronizado }\end{array}$ & $t$ & $p$ \\
\hline Constante & 2,025 & 0,180 & & 11,251 & $0,000^{*}$ \\
\hline Primeiro Emprego & 0,556 & 0,185 & 0,238 & 3,003 & $0,003^{*}$ \\
\hline Classe Econômica & 0,485 & 0,185 & 0,208 & 2,627 & $0,010^{*}$ \\
\hline Sexo & 0,255 & 0,175 & 0,113 & 1,457 & 0,147 \\
\hline \multicolumn{6}{|c|}{ 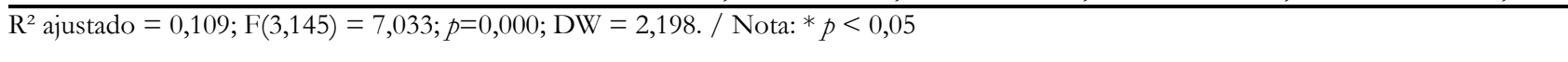 } \\
\hline \multicolumn{6}{|c|}{ Bloco 2} \\
\hline Variáveis preditivas & Beta & $\begin{array}{c}\text { Erro } \\
\text { Padrão }\end{array}$ & $\begin{array}{c}\text { Beta } \\
\text { padronizado }\end{array}$ & $t$ & $p$ \\
\hline Constante & 1,936 & 0,191 & & 10,150 & $0,000^{*}$ \\
\hline Primeiro Emprego & 0,554 & 0,184 & 0,237 & 3,007 & $0,003^{*}$ \\
\hline Classe Econômica & 0,475 & 0,183 & 0,204 & 2,592 & $0,011 *$ \\
\hline Sexo & 0,245 & 0,174 & 0,109 & 1,407 & 0,162 \\
\hline Condição de Deficiência & 0,200 & 0,174 & 0,089 & 1,149 & 0,252 \\
\hline
\end{tabular}

$\mathrm{R}^{2}$ ajustado $=0,114 ; \mathrm{F}(4,145)=8,808 ; p=0,000 ; \mathrm{DW}=2,175 . /$ Nota: $* p<0,05$ 
Quando a variável condição de deficiência é inserida, verifica-se um pequeno acréscimo no poder explicativo do modelo $\left(\mathrm{R}^{2}\right.$ ajustado $\left.=0,114\right)$. $\mathrm{O}$ teste de Kolmogorov-Smirnov apresentou, respectivamente, bloco 1 com $p=0,437$; bloco 2 com $p=0,469$, confirmando a hipótese de normalidade dos resíduos, o que ratifica os resultados da regressão.

\section{Discussão}

Segundo dados da RAIS (MTE, 2010), houve uma ligeira elevação na força de trabalho feminina $(41,6 \%)$, refletindo a expansão da mulher no mercado de trabalho. Porém, no que se refere às $\mathrm{PcD}$, o gênero masculino $(65,42 \%)$ é o que possui maior representatividade na área trabalhista. Essas informações se assemelham aos dados sociodemográficos dos respondentes deste estudo, visto que $56 \%$ da amostra é constituído por homens. No que se refere à idade, a média dos entrevistados é de 32 anos, corroborando também o estudo de Abreu (2011) e a pesquisa da RAIS (MTE, 2010), que indicaram um maior incremento de empregos gerados na faixa etária de 30 a 39 anos.

Embora pesquisas anteriores evidenciem o baixo nível de escolaridade das $\mathrm{PcD}$ e por essa razão uma dificuldade maior em inseri-los no mercado de trabalho (Ribeiro \& Carneiro, 2009), dados deste estudo indicaram que a maior parte das pessoas entrevistadas (pessoas com e sem deficiência) possuía o ensino médio completo - 41,3\% e 49,3\%, respectivamente -, seguido pelo ensino superior completo $(14,7 \%$ e $13,3 \%$, respectivamente). No que se refere à remuneração média entre os dois grupos, não existe diferença significativa entre os mesmos - média salarial das $\mathrm{PcD}$ : $2,32 \%$, o que corresponde a $\mathrm{R} \$ 1.253,50$; e média salarial das PsD: $2,57 \%$, equivalente a $\mathrm{R} \$ 1.417,00$.

Foi possível constatar que não existe diferença significativa entre os resultados de comprometimento organizacional e satisfação com fatores de QVT entre as $\mathrm{PcD}$ e as $\mathrm{PsD}$ que realizam o mesmo trabalho. Todas elas estabelecem diferentes tipos de comprometimento com a organização da qual fazem parte, sem, contudo, a presença de um desses tipos eliminar a possibilidade da manifestação de outro. Verificou-se que o comprometimento afetivo, que se refere a um maior envolvimento do indivíduo com a organização de trabalho, é o predominante entre as pessoas com e sem deficiência, fato que ratifica resultados de pesquisa anterior realizada com pessoas com deficiência (Almeida e cols., 2009). Por outro lado, níveis reduzidos de comprometimento normativo indicam que tanto as $\mathrm{PsD}$ quanto as $\mathrm{PcD}$ não se sentem na obrigação moral de permanecer na organização em que trabalham, confirmando outros estudos (Almeida e cols., 2009) e ratificando a dúvida de muitos gestores que não conseguem identificar a existência de diferenças entre o comprometimento de pessoas com e sem deficiência (Carvalho-Freitas, 2009). Já em relação ao baixo índice de comprometimento instrumental, este pode estar associado às possibilidades oriundas da Lei de Cotas e aos baixos índices de desemprego nas regiões durante a realização da pesquisa, o que minimiza a necessidade de se permanecer em empregos porque precisam deles.

Além disso, os resultados das correlações demonstraram que todos os fatores de satisfação relacionados à QVT estão correlacionados positivamente com o comprometimento afetivo, o que confirma os resultados de outras pesquisas que indicam que esses dois construtos são correlatos (Mathieu \& Zajac, 1990; Meyer e cols., 2002; Siqueira \& Gomide Jr., 2004). Também o comprometimento normativo se apresentou correlacionado com todos os fatores de satisfação. Por outro lado, o comprometimento instrumental se apresentou correlacionado negativamente com a integração social e a relevância do trabalho. Esses resultados sugerem que as pessoas que estão satisfeitas com as oito dimensões de trabalho relacionadas à QVT (remuneração, condição de trabalho, possibilidade de uso e desenvolvimento das capacidades, possibilidade de crescimento, integração social, direitos, equilíbrio trabalho e vida, e relevância do trabalho) tendem também a manter níveis mais altos de comprometimento afetivo e normativo. Por outro lado, as pessoas insatisfeitas com a integração social e com a relevância do trabalho tendem a ter um maior comprometimento instrumental, isto é, permanecem na organização porque precisam daquele emprego. Esses resultados representam indicações importantes para a prática de gestão de pessoas nas organizações, pois demonstram que $\mathrm{O}$ investimento nos fatores relacionados à QVT terá impacto no compromisso e no desejo das pessoas se manterem na organização, o que corrobora os resultados da literatura sobre comprometimento (Mathieu \& Zajac, 1990; Meyer e cols., 2002; Siqueira \& Gomide Jr., 2004). No entanto, difere de pesquisa realizada com pessoas com deficiência que atuavam no setor bancário em que o comprometimento normativo estava correlacionado apenas com a remuneração e o comprometimento instrumental não se correlacionou com nenhum fator de satisfação no trabalho (Carvalho-Freitas e cols., 2009). Novas pesquisas serão necessárias para verificar possíveis diferenças nesses resultados.

Os resultados das análises de regressão indicaram que a variância do comprometimento afetivo não pode ser explicada por fatores sociodemográficos. No 
entanto, verificou-se que a variabilidade nos resultados do comprometimento normativo pode ser explicada por algumas características pessoais, isto é, as pessoas que estão no emprego entre um e cinco anos, quando comparadas com trabalhadores novatos (menos de um ano de trabalho), têm menor comprometimento normativo. Além disso, as pessoas de menor renda familiar (até três salários mínimos) têm maior comprometimento normativo que as de classe econômica com renda superior a três salários mínimos. Esses resultados indicam que as pessoas de menor renda familiar e que estão a menos de um ano no emprego se sentem mais gratas à organização e com o dever moral de permanecer nela. Esses resultados, tanto em relação ao comprometimento afetivo (que não apresentou relação de dependência com características pessoais) quanto ao normativo confirmam os achados de estudos anteriores (Mathieu \& Zajac, 1990; Meyer e cols., 2002). O presente estudo incluiu a variável condição de deficiência e verificou que ela também não contribui para explicar a variação nos comprometimentos afetivos e normativos, ratificando indicações de pesquisa anterior realizada com pessoas com deficiência (Carvalho-Freitas e cols., 2009).

Em relação ao comprometimento instrumental, verificou-se, com base nas análises de regressão, que tanto fatores sociodemográficos quanto a condição de deficiência explicaram a variabilidade de seus resultados. Foi demonstrado que as pessoas que estão em seu primeiro emprego, quando comparadas às demais; que as pessoas que têm menor renda familiar (até três salários mínimos), quando comparadas às de maior renda familiar; e que as pessoas do sexo masculino, quando comparadas com as do sexo feminino, têm maior comprometimento instrumental. Esse resultado ratifica pesquisas anteriores que indicam que as características pessoais são antecedentes do comprometimento instrumental (Mathieu \& Zajac, 1990; Meyer e cols., 2002). Além disso, quando se incluiu a condição de deficiência na análise de regressão, verificou-se que as pessoas com deficiência, nessas mesmas condições de primeiro emprego, renda familiar menor e do sexo masculino, têm maior comprometimento instrumental que as pessoas sem deficiência. Esse resultado, não identificado em pesquisas anteriores, indica que as pessoas com deficiência, com esse perfil, tendem a se manter nas organizações porque consideram que precisam do trabalho e que teriam menos oportunidades de conseguir outros empregos quando comparadas às pessoas sem deficiência. No entanto, essas $\mathrm{PcD}$ não investem na organização mais do que o necessário para fazer o trabalho, característica desse tipo de comprometimento, segundo Rego, Cunha e Souto (2007).

Os resultados da presente pesquisa têm um impacto importante nas investigações sobre a inserção de pessoas com deficiência, porque indicam que quando as pessoas estão satisfeitas com fatores de contexto de trabalho (QVT), elas, independentemente de terem ou não uma deficiência, têm maior comprometimento afetivo com a organização. Esse tipo de comprometimento, segundo a literatura (Siqueira \& Gomide Jr., 2004) tem como consequência melhor desempenho, maior esforço no trabalho, menor rotatividade, menos faltas e atrasos. Além disso, os resultados indicaram que não existe diferença significativa no que se refere ao comprometimento normativo de pessoas com e sem deficiência em circunstâncias similares de trabalho. Esse resultado indica que não é verdadeira a premissa de alguns gestores (Carvalho-Freitas, 2009, Carvalho-Freitas, \& Marques, 2010) de que as pessoas com deficiência permanecem nas organizações porque se sentem gratas à oportunidade que lhes foi concedida. Esse comprometimento (normativo) está correlacionado com a satisfação com fatores de QVT. Esses resultados somam-se ao de pesquisa anterior realizada por Pereira, Del Prette A. e Del Prette Z. (2008) que verificaram que a deficiência física não pode ser considerada um fator determinante no significado atribuído ao trabalho.

\section{Considerações finais}

Os resultados desta pesquisa permitiram verificar que, garantida a satisfação com fatores relacionados ao contexto de trabalho, as PcD têm resultados de comprometimento afetivo e normativo similares aos das demais pessoas. No entanto, quando possuir uma deficiência se associa a condições sociais de desvantagem (menor renda familiar e primeiro emprego) e ao fato de ser do sexo masculino, as pessoas com deficiência tendem a ter um maior comprometimento instrumental, isto é, elas tendem a permanecer no trabalho porque avaliam que precisam dele, mais do que ocorre entre pessoas sem deficiência.

Esses resultados empíricos ratificam a tendência dos estudos internacionais em indicar que é necessária uma mudança no foco das pesquisas sobre PcD, buscando identificar as barreiras e os impactos ambientais e sociais na produção de desvantagens para as $\mathrm{PcD}$ (Barnes, Oliver, \& Barton, 2002; Finkelstein, 1980; Oliver, 1996; WHO, 2011). É necessário construir uma agenda de pesquisas, buscando identificar fatores que interferem na adaptação das $\mathrm{PcD}$ nos diversos espaços sociais e suas repercussões para essas pessoas. Novas pesquisas com amostras de 
diferentes partes do país poderiam ampliar as constatações desta pesquisa e contribuir para a construção de políticas organizacionais que contemplem melhor a questão da diversidade nos ambientes de trabalho.

\section{Referências}

Abreu, M. V. (2011). A qualidade de vida no trabalho de pessoas com deficiência: um estudo de caso em uma grande empresa do setor metalírgico (Dissertação de Mestrado). Pedro Leopoldo: Centro de PósGraduação - Faculdades Pedro Leopoldo.

Allen, N. \& Meyer, J. P. (1996). Affective, continuance, and normative commitment to the organization: An examination of construct validity. Journal of Vocational Behavior, 49, 252-276.

Almeida, L. A. D., Carvalho-Freitas, M. N., \& Marques, A. L. (2009). Inserção no mercado formal de trabalho: satisfação e condições de trabalho sob olhar das pessoas com deficiência. Em M. N. Carvalho-Freitas, M. N. \& Marques, A. L. (Orgs.). Trabalho e pessoas com deficiência: pesquisas, práticas e instrumentos de diagnóstico. (p. 89105)Curitiba: Juruá.

Barbosa-Gomes, J. F. (2009). Contribuiçoes da categoria trabalho na formação da identidade da pessoa com deficiência (Dissertação de Mestrado). Pontifícia Universidade Católica de Minas Gerais.

Barnes, C., Oliver, M., \& Barton, L. (2002). Disability studies today. Cambridge: Polity.

Bastos, A. V. B. (1994). Comprometimento organizacional: a estrutura dos vinculos do trabalhador com a organização, a carreira e o sindicato (Tese de Doutorado). Instituto de Psicologia - Universidade de Brasília.

Bastos, A. V. B., Brandão, M. G. G., \& Pinho, A. P. M. (1997). Comprometimento organizacional: uma análise do conceito expresso por servidores universitários no cotidiano de trabalho. RAC Revista de Administração Contemporânea, 1(2), 97-120.

Butterfield, T. M., \& Ramseur, J. H. (2004). Research and case study findings in the area of workplace accommodations including provisions for assistive technology: a literature review. Technology and Disability, 16(4), 201-210.

Carvalho-Freitas, M. N. (2007). A inserção de pessoas com deficiência em empresas brasileiras (Tese de Doutorado). Centro de Pós-Graduação e Pesquisa em Administração - Universidade Federal de Minas Gerais.

Carvalho-Freitas, M. N. (2009). Inserção e gestão do trabalho de pessoas com deficiência: um estudo de caso. RAC Revista de Administração Contemporânea, 13(edição especial), 121-138.
Carvalho-Freitas, M. N., \& Marques, A. L. (2009). Trabalho e pessoas com deficiência: pesquisas, práticas e instrumentos de diagnóstico. Curitiba: Juruá.

Carvalho-Freitas, M. N., \& Marques, A. L. (2010). Inserção de pessoas com deficiência em empresas brasileiras: um estudo com empresas socialmente responsáveis. Revista Eletrônica de Gestão Organizacional, 8(3), 483-502.

Carvalho-Freitas, M. N., Marques, A. L., \& Almeida, L. D. (2009). Pessoas com deficiência: comprometimento organizacional, condições de trabalho e qualidade de vida no trabalho. Gerais: Revista Interinstitucional de Psicologia, 2(2), 92-105.

Finkelstein, V. (1980). Attitudes and disabled people: issues for discussion. International exchange of information in rehabilitation. New York, USA.

Gartrell, A. (2010). A frog in a well: the exclusion of disabled people from work in Cambodia. Disability and Society, 25(3), 289-301.

Hackman, J. R., \& Oldham, G. R. (1975). Devolopment of the job diagnostic survey. Journal of Applied Psychology, 60(2), 159-170.

Honório, L. C., Marques, A. L., \& Melo, M. S. (2001). Qualidade de vida no trabalho em uma micro empresa do comércio varejista. Encontro da Associação Nacional dos Programas de Pós-Graduação em Administração, Campinas, SP, Brasil.

Instituto Brasileiro de Geografia e Estatística. (2010). Censo de 2010. Recuperado em 20 agosto, 2011, de http://www.censo2010.ibge.gov.br/index.php

Instituto Ethos. Perfil social, racial e de gênero das 500 maiores empresas do brasil e suas ações afirmativas. Recuperado em 20 junho, 2011, de http://www1.ethos.org.br/EthosWeb/arquivo/0A-eb4Perfil_2010.pdf.

Jolly, D. (2000). A critical evaluation of the contradictions for disabled workers arising from the emergence of the flexible labour market in Britain. Disability and Society, 15(5), 795-810.

Maia, L. M., Camino, C., \& Camino, L. (2011, janeiro/julho). Pessoas com deficiência no mercado de trabalho: uma análise do preconceito a partir das concepções de profissionais de Recursos Humanos. Pesquisas e Práticas Psicossociais, 6(1), 7891.

Martins, M. C. F., \& Santos, G. E. (2006, julho/dezembro). Adaptação e validação de construto na escala de satisfação no trabalho. PsicoUSF, 11(2), 195-205.

Mathieu, J. E., \& Zajac, C. (1990). A review and metaanalysis of the antecedentes, correlates, and consequences of organizational commitment. Psychological Bulletin, 108, 171-194. 
Meyer, J. P., Stanley, D. J., Herscovitch, \& Topolnytsky, L. (2002). Affective, continuance and normative commitment to the organization: a meta-analysis of antecedents, correlates and consequences. Journal of Vocational Behavior, 61, 2052.

Ministério do Trabalho e Emprego. (2010). Características do emprego formal segundo a Relação Anual de Informaçoes Sociais - 2010. Recuperado em 20 outubro, 2011, de http://www.mte.gov.br/rais/2010/arquivos/Resu ltados_Definitivos.pdf.

Nascimento, L. C., Damasceno, G. J. B., \& Assis, L. J. (2011, janeiro/julho). Mercado de trabalho para as pessoas com deficiência em Betim/MG. Pesquisas e Práticas Psicossociais, 6(1), 92-101.

Oliver, M. (1996). Understanding disability: form theory to practice. New York: Palgrave.

Opini, B.,M. (2010). A review of the participation of disabled persons in the labour force: the Kenyan context. Disability and Society, 25(3), 271-287.

Pereira, C. S., Del Prette, A., \& Del Prette, Z. A. P. (2008, janeiro/junho). Qual o significado do trabalho para as pessoas com e sem deficiência física? Psico-USF, 13(1), 105-114.

Rego, A., Cunha, M. P., \& Souto, S. (2007). Espiritualidade nas organizações e comprometimento organizacional. RAE-eletrônica, 6(2). Artigo 12, 1-27.

Ribeiro, M. A., \& Carneiro, R. (2009, julho/setembro). A inclusão indesejada: as empresas brasileiras face à Lei de Cotas para pessoas com deficiência no mercado de trabalho. Organizacõos \& Sociedade, 16(50), 545-564.

Rodrigues, A. C. A., \& Bastos, A. V. B. (2009). Problemas conceituais e empiricos na pesquisa sobre comprometimento organizacional: uma análise crítica do modelo tridimensional de J. Meyer e N. Allen. Encontro Anual da Associação Nacional de Pós-Graduação e Pesquisa em Administração, São Paulo, SP, Brasil, 33.

Shier, M, Grahan J., \& Jones M. E. (2009). Barriers to employment as experienced by disabled people: a qualitative analysis in Calgary and Regina. Canadá. Disability and Society, 24(1), 63-75.

Siqueira, M. M. M. (2002). Esquema mental de reciprocidade $e$ influências sobre afetividade no trabalho. Encontro Anual da Associação Nacional de Pós-Graduação e Pesquisa em Administração, Salvador, BA, Brasil.
Siqueira, M. M. M., \& Gomide Júnior, S. (2004). Vínculos do indivíduo com o trabalho e com a organização. Em J. C. Zanelli, J. E. BorgesAndrade, \& A. V. B. Bastos (Orgs.). Psicologia, Organizaçoes e Trabalho no Brasil. (pp. 300-330) Porto Alegre: Artmed.

Stone, D. L., \& Colella, A. (1996). A model of factors affecting the treatment of disabled individuals in organizations. Academy of Management Review. 21, 96-104.

Stone-Romero, E. F., Stone, D. L., \& Lukaszewski, K. (2006). The influence of disability on role-taking in organizations. Em A. M. Konrad, P. Prasad, \& J. K. Pringle (Orgs). Handbook of workplace diversity (pp. 401-430). Thousand Oaks: Sage.

Suzano, J. C. C. E cols. (2009). Análise da produção acadêmica nacional dos últimos 20 anos sobre a inserção da pessoa portadora de deficiência no mercado de trabalho. Em M. N. Carvalho-Freitas \& A. L. Marques. Trabalho e pessoas com deficiência: pesquisas, práticas e instrumentos de diagnóstico. (pp. 2342)Curitiba: Juruá.

Tanaka, E. D. O., \& Manzini, E. J. (2005). O que os empregadores pensam sobre o trabalho da pessoa com deficiência? Revista Brasileira de Educação Especial, 11(2), 273-294.

Vasconcelos, A. F. (2001). Qualidade de vida no trabalho: origem, evolução e perspectiva. Cadernos de Pesquisas em Administração, 8(1), 21-29.

Walton, R. (1973). Quality of working life: what is it? Sloan Management Review, 1(1), 11-21.

Wehbi, S. \& El-Lahib Y. (2007). The employment situation of people with disabilities in Lebanon: challenges and opportunities. Disability and Society, 22(4), 371-382.

World Health Organization. (2011). World report on disability. Recuperado em 2 dezembro, 2011, de http://whqlibdoc.who.int/publications/2011/978 9240685215_eng.pdf.

Zolna, J. S., Sanford, J., Sabata, D., \& Goldthwaite, J. (2007). Review of accommodation strategies in the workplace for persons with mobility and dexterity impairments: application to criteria for universal design. Technology and Disability, 19(4), 189-198.

Recebido em 20/03/2012

Reformulado em 06/03/2013

Aprovado em 20/03/2013 
Nota dos autores:

Agradecemos à FAPEMIG e ao CNPq pelo apoio ao financiamento da pesquisa.

Sobre os autores:

Maria Nivalda de Carvalho-Freitas é psicóloga, doutora em Administração pela Universidade Federal de Minas Gerais, professora adjunta do Departamento de Psicologia e professora do Programa de Pós-Graduação Stricto Sensu em Psicologia da Universidade Federal de São João del-Rei. Coordena o Núcleo de Pesquisas em Acessibilidade, Diversidade e Trabalho - NACE.

Liliam Medeiros da Silva é psicóloga pela Universidade Federal de São João Del-Rei - UFSJ. Atualmente é mestranda do Programa de Pós-Graduação Stricto Sensu em Psicologia da Universidade Federal de São João del-Rei e membro do Núcleo de Pesquisa em Acessibilidade, Diversidade e Trabalho - NACE. Realiza estudos na linha de pesquisa "Processos Psicossociais e Sócio-Educativos", especificamente na inserção social de pessoas com deficiência.

Suelen Priscila Macedo Farias é graduanda do curso de Psicologia pelas Faculdades Integradas Pitágoras de Montes Claros - FIPMoc, membro da Associação Norte Mineira de Apoio ao Autismo - ANDA e membro do Núcleo de Apoio a Inclusão Pitágoras - NAIP. Atua nos seguintes temas: autismo, educação especial e inclusão da pessoa com deficiência no ensino superior.

Marcos Santos de Oliveira é estatístico pela Universidade Estadual Paulista, mestre em Estatística pela Universidade de São Paulo e doutor em Estatística e Experimentação Agropecuária pela Universidade Federal de Lavras. Atualmente é professor adjunto do Departamento de Matemática e Estatística e docente colaborador no Programa de Pós-Graduação Stricto Sensu em Psicologia da Universidade Federal de São João del-Rei.

Raissa Pedrosa Gomes Tette é psicóloga pela Universidade Federal de São João Del Rei - UFSJ. Atualmente é mestranda do Programa de Pós-Graduação Stricto Sensu em Psicologia da Universidade Federal de São João del-Rei e membro do Núcleo de Pesquisa em Acessibilidade, Diversidade e Trabalho - NACE. Realiza estudos na linha de pesquisa "Processos Psicossociais e Sócio-Educativos", especificamente na inserção social de pessoas com deficiência.

Contato com os autores:

Universidade Federal de São João del-Rei

Rua Felipe Marchetti, 80 - CEP 36307-248 - Vila Marchetti - São João del-Rei/MG, Brasil.

E-mail: nivalda@ufsj.edu.br 